\title{
Fifty years of pheromones
}

\section{Powerful chemical signals have been identified in moths, elephants and fish, recounts Tristram D. Wyatt. But, contrary to stories in the popular press, the race is still on to capture human scents.}

Fifty years ago this month, Peter Karlson and Martin Lüscher proposed a new word for the chemicals used to communicate between individuals of the same species: pheromones ${ }^{1}$. Since then, pheromones have been found across the animal kingdom, sending messages between courting lobsters, alarmed aphids, suckling rabbit pups, mound-building termites and trail-following ants. They are also used by algae, yeast, ciliates and bacteria.

The new word met a pressing need. Karlson had discussed it with his colleague Adolf Butenandt, who was about to publish the first chemical identification of a pheromone bombykol, the sex pheromone of the silk moth Bombyx mori. The bombykol paper showed the equivalent of Koch's postulates for establishing causal relationships for pheromones: isolation, identification, synthesis and bioassay confirmation of activity ${ }^{2}$. Butenandt's work established that chemical signals between animals exist and can be identified, marking the start of modern pheromone research. Popular speculation about human pheromones, still going strong today, began too.

The idea of chemical communication was not new in 1959. The ancient Greeks knew that the secretions of a female dog in heat attracted males. Charles Butler had warned in The Feminine Monarchie (1609) that if you are stung by one honeybee, "other Bees smelling the ranke favour of the poison cast out with the sting will come about you as thick as haile". In The Descent of Man, and Selection in Relation to Sex (1871), Charles Darwin included chemical signals alongside visual and auditory ones as outcomes of sexual selection, describing the success of the smelliest among breeding male crocodiles, ducks, goats and elephants. JeanHenri Fabre, also in the 1870 s, described how male emperor moths flocked around a female moth hidden behind wire-gauze, but ignored visible females sealed under glass. Surely her smell was the attraction.

In 1932, the physiologist Albrecht Bethe had proposed the broad term 'ectohormone' to cover many kinds of chemical interaction, including communication or attraction of an animal to a food smell. Karlson and Lüscher wanted a term that more narrowly covered communication between members of the same species, but more broadly allowed for those chemicals to be created by a variety of organs ('hormones' by definition come from the endocrine glands). Their new term, from the Greek pherein for 'to transfer', and hormōn ' to excite', at a stroke replaced ectohormone. 'Pheromone' was sonorous, and close enough to 'hormone' to imply some similarities along with the differences: like hormones, pheromones could be expected to be specific, and active in minute amounts. They defined pheromones as: "substances which are secreted to the outside by an individual and received by a second individual of the same species, in which they release a specific reaction, for example, a definite behaviour or a developmental process." The new word and definition stuck.

\section{Feast for the senses}

Karlson and Lüscher were far-sighted, noting that pheromones were likely to be used by a wide range of animals, including fish and underwater crustaceans as well as land mammals and insects. They predicted that most pheromones would act via the conventional senses of smell or taste, but that some might be ingested and act directly on the brain or other organs - as happens in termites, whose pheromones affecting caste development are passed round by mouth through the colony.

All these anticipations have been borne out, although Karlson and Lüscher might have been amazed at the range of molecules identified as pheromones since 1959 , including everything from low-molecularweight formic acid to polypeptides. We now know that many pheromones (including the sex pheromones of most moths) are not single compounds, but rather a species-specific combination of molecules in a precise ratio.

The ubiquity and variety of pheromones can be explained by natural selection. The evolutionary development of sex pheromones in a fish, for example, might have started with male fish detecting sex hormones leaking from a female about to spawn. The most sensitive males would get there first. Over generations, there would be selection for increased sensitivity of the receiver and increased production of the signal by the sender.

Chemical communication can also be exploited by other species. For example, some orchids, which benefit from attracting pollinators, produce a mixture of compounds that mimics female-wasp pheromones. The mimicry is so good that duped males will ejaculate on the flowers.

Karlson also catalysed a completely new field of study in biology, by asking a young biologist neighbour, Dietrich Schneider, if he could invent an electrophysiological way to assess Butenandt's silk-moth extracts for activity. Schneider's solution was the electroantennogram, still used today: wires inserted into both ends of a moth antenna are used to measure electrical signals as different extracts are presented. Recordings of activity in single antennal sensory cells followed in later years, as moths and their pheromones became a key model system in neurobiology.

The pursuit of pheromone science has not been entirely sweet and easy. The concept has faced key periods of controversy over mammalian pheromones, in battles almost as heated as the 'stink wars' between opposing troops of ring-tailed lemurs, which wave their pheromone-coated tails to assert their dominance.

In the 1970s, a group of researchers studying mammals argued that the term 'pheromones' should not be used for mammalian chemical signals, citing in particular the complex, highly variable odours that mammals use to distinguish littermate from stranger, for example for altruism or mate choice. These individual odours, including some related to the immune system, need to be learnt for recognition, and did not seem to fit Karlson and Lüscher's definition. Some researchers even doubted that complex mammals, including humans, could have their behaviour altered by something as simple as an instinctive reaction to a smell.

Debate continues among those in the field. I now agree that these variable odours are not pheromones, and instead are better termed 'signature odours' (the same holds for complex variable odours in social insects such as ants and bees, which also have to be learnt and are used for colony recognition). But speciesspecific small molecules that do fit the classic pheromone definition have now been identified for mammals. Most spectacular was the 1996 discovery that the female Asian elephant's sex pheromone is a small molecule - $(Z)-7$ dodecen-1-yl acetate - also used by some 


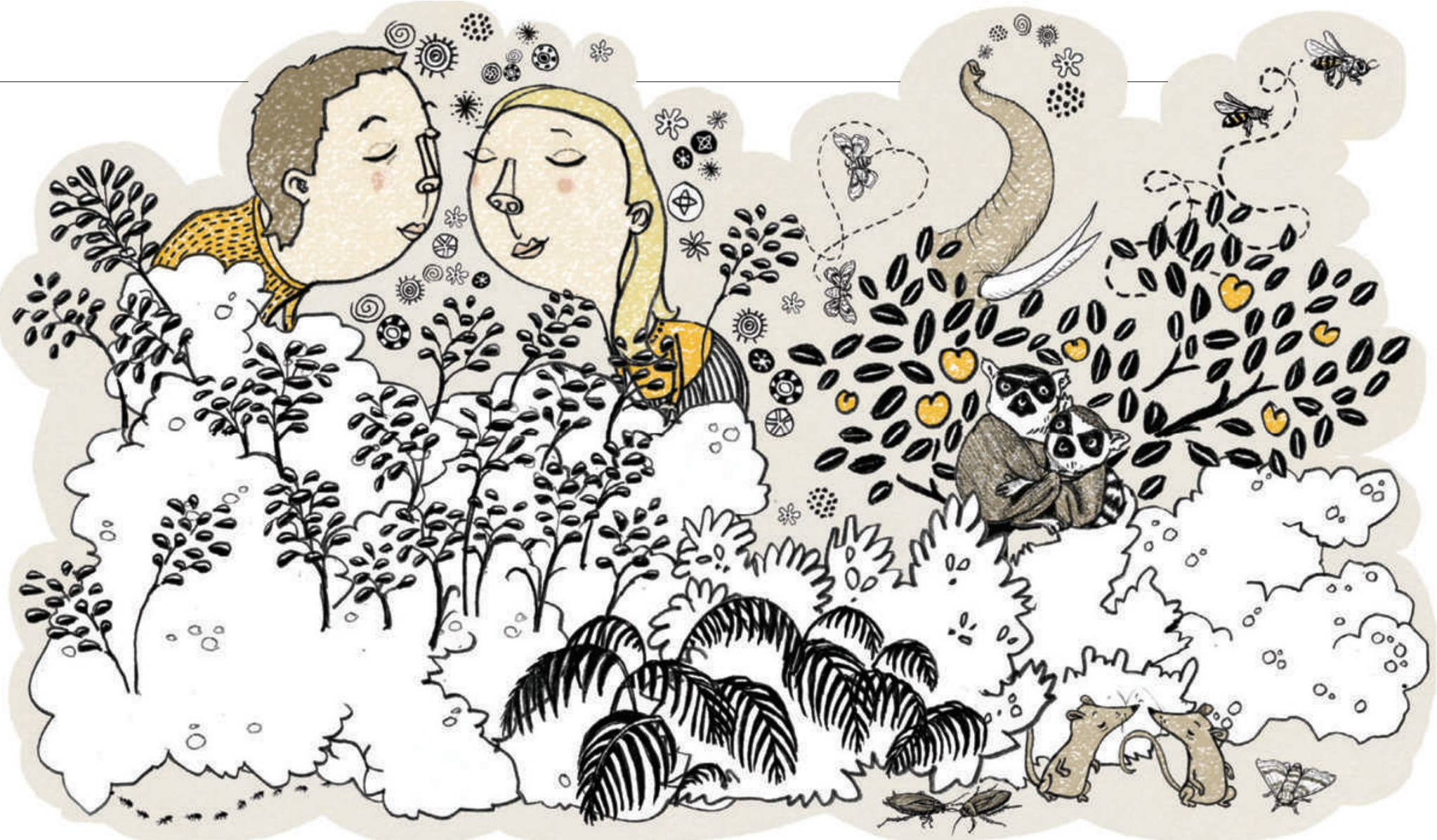

140 species of moth as a component of their female sex pheromones. Signature odours and pheromones can be mixed. Some mammals, including elephants and mice, present their small-molecule pheromones in the cleft of highly variable urinary (or other lipocalin) proteins. As well as overlaying the anonymous pheromone signals with individual signature odours, the proteins release the small molecules slowly, making them last longer.

\section{Person-to-person}

A second group of researchers - mainly molecular biologists - accepted the idea that mammals have pheromones, but proposed that these are exclusively detected by a specialized sensory system, the vomeronasal organ (VNO), rather than the main olfactory epithelium in the nose. This view perhaps arose because some pheromones in mice do act via the $\mathrm{VNO}$, and the mouse is a widely used model. As humans do not have a VNO, this theory would exclude them from sensing and responding to pheromone signals.

This argument persisted despite extensive evidence from behavioural studies and neurobiologists that information from the $\mathrm{VNO}$ and the main olfactory system was integrated in the rodent brain, and that some mammal pheromones, such as the rabbit mammary pheromone, acted via the nose. Finally, in
2005, a series of molecular studies in mice, using combinations of genetic markers and knockouts, confirmed that pheromones can stimulate both systems, which are integrated in the brain. So humans wouldn't need a VNO to receive pheromone signals after all.

The identification and synthesis of pheromones have been put to good use, giving the greenest way to control pests ranging from moths in cotton fields to lampreys in the Great Lakes of North America. Pheromones can be used to lure pests into a death trap or confuse males so that they never find a female; pheromones' high specificity and low toxicity leave ecosystems intact.

Advances in analytical techniques have enabled progress. Butenandt's team needed 500,000 female silk moths to produce 12 milligrams of material for the first pheromone identification. Today, one moth might do. New tools will power new discoveries. For example, we can now follow the chemical signals emitted by fighting wasps, in real time. Microarray genomics shows genes switching on and off in the honeybee brain in response to pheromones. But challenges remain. Correct synthesis of pheromone molecules is harder than their identification. We can record and play back the audible calls of an animal easily enough, but we don't have a video or MP3 equivalent for recreating chemical signals.

What about humans? As we're mammals, we are likely to use pheromones. Our armpits are prime candidates as sources, as their smells develop along with other changes at puberty. However, both human behaviour and our chemical emissions are so complex that the research is challenging: so far, no pheromones have been conclusively identified, despite stories in the popular press. A strong contender for the first real human pheromone is some compound in women's armpit extract that apparently causes menstrual synchrony

in females living in close quarters. Its identification is keenly awaited, not least as it could potentially open the door to sniffable contraceptives. There may never be a magic potion to make us irresistible, but I'm sure human pheromones will surprise us yet.

Tristram D. Wyatt is in the Department of

Zoology of the University of Oxford, UK, and is the author of Pheromones and Animal Behaviour. e-mail: tristram.wyatt@zoo.ox.ac.uk

\footnotetext{
Karlson, P. \& Lüscher, M. Nature 183, 55-56 (1959).

2. Butenandt, A., Beckmann, R., Stamm, D. \& Hecker, E.

Z. Naturforsch. B 14, 283-284 (1959).
}

See http://tinyurl.com/9bll5d for further reading. 